\title{
Introduction and Safety Evaluation of Citrinin in Foods
}

\author{
Wang Huiqin, Zhang Shufen, Lin Taifeng, Zheng Dawei \\ College of Life Science \& Bioengineering, Beijing University of Technology, Beijing, China
}

\section{Email address:}

wanghuiqin@bjut.edu.cn (Wang Huiqin)

\section{To cite this article:}

Wang Huiqin, Zhang Shufen, Lin Taifeng, Zheng Dawei. Introduction and Safety Evaluation of Citrinin in Foods. Journal of Food and Nutrition Sciences. Vol. 5, No. 5, 2017, pp. 179-183. doi: 10.11648/j.jfns.20170505.13

Received: June 29, 2017; Accepted: July 20, 2017; Published: August 7, 2017

\begin{abstract}
Citrinin is a kind of mycotoxin, which is produced during Monascus fermentation to produce Monascus Pigments. Studies have shown that citrinin has certain nephrotoxicity and hepatotoxicity, which can cause tumor, induce mutation and so on. The pretreatment of samples was carried out by ultrasonic assisted extraction centrifugal separation and nitrogen blowing concentration, and HPLC with fluorescence detection was used to detect 4 types samples of food raw materials including Monascus food additives (a total of 57 samples). The results showed that citrinin was 23 positive and the positive rate was $40.4 \%$ (Below the detection limit is negative, opposite positive). The pollution level reached $0.096 \sim 0.24 \mathrm{mg} / \mathrm{kg}$ and the average pollution level is $0.16 \mathrm{mg} / \mathrm{kg}$. The problem of citrinin should be paid enough attention to in the Monascus production industry, and it may be possible to solve the problem of citrinin in Monascus by biological, physical or chemical methods.
\end{abstract}

Keywords: Citrinin, Monascus, Safety Evaluation

\section{Introduction}

Over thousands of years, Monascus pigments have been widely used traditionally in food products and pharmaceutical industries, e.g. Chinese cheese, meat, bagoong, wine, tofu, miso and fish [1]. In the food industry, Monascus can be fermented to produce red rice, red wine, red bean curd and other traditional foods; however, the safety of Monascus has been challenged, because in the process of fermentation of most Monascus will produce a variety of functional material, simultaneously can produce mycotoxins, such as citrinin. It has been regarded as an important index of food contamination abroad. Japan pioneered the formulation of citrinin limits in Monascus pigments, The U.S. FDA has made it clear that Monascus products are recognized as food additives and must be evaluated for citrinin. Europe and the United States have developed strict limits on citrinin in Monascus; however, our country has not formulated the limit standard of citrinin in Monascus at present. It is necessary to investigate citrinin in the market using Monascus pigments in order to provide the basis for establishing the limited standard as early as possible.

\section{Introduction of Citrinin}

Citrinin is a kind of mycotoxin, which is produced during metabolism by molds such as Penicilliu, Aspergillus, and Monascus. It was isolated from Monascus for the first time by Hinchumg Wang in 1981, and its structural characteristics were confirmed by absorption spectra, MS and NMR by French scholars Blanc and so on in 1995.

\subsection{Physical and Chemical Properties of Citrinin [2]}

The molecular formula of citrinin (or penicillin citrinin) is $\mathrm{C}_{13} \mathrm{H}_{14} \mathrm{O}_{5}$, relative molecular weight: 250 . The chemical nomenclature is [(3 R, $4 \mathrm{~S})-4,6$-dihydrogen-8-hydroxyl-3, 4, 5-trimethyl-6-oxygen-3 H-2-Phenylpyrazole-7-carboxylic acid], which has two isomers as follows (figure 1).<smiles>CC1=C2C(=COC1C)C(O)=C(C(=O)O)C(=O)C2C</smiles><smiles></smiles>

Figure 1. The molecular structure of citrinin.

As an acidic substance citrinin is a lemon yellow crystalline substance with a melting point of $172^{\circ} \mathrm{C}$ at room temperature. Under the excitation of long wave ultraviolet lamp, citrinin can emit yellow fluorescence. Its maximum UV absorption 
wavelength is $250 \mathrm{~nm}$ and $333 \mathrm{~nm}$ in methanol solution. Citrinin is difficult to dissolve in water, but its sodium salt is soluble in water and soluble in chloroform, acetone, ethyl acetate, ethanol and most other polar organic solvents. Citrinin can form chelates and can degrade in acidic or alkaline solutions and degrade in heating.

\subsection{Production of Citrinin}

Monascus has a long history of application in China and has passed toxicological experiments. However, the discovery of citrinin casts a shadow on the ancient fine strain of monascus. Citrinin is associated with the production of Monascus Pigments. Monascus has nearly a thousand years of history in China which can produce many kinds of traditional foods, such as red yeast rice, red wine, red Sufu and so on. But as a mycotoxin citrinin harmful to humans and animals, citrinin was detected in the culture of Monascus by the French scholar Blanc for the first time in 1995. This discovery caused a lot of vibration in the food industry, which attracted the attention of some experts and scholars to study the issue of citrinin produced by monascus. At the same time, it also limits the application of Monascus products in the world.

The partial metabolic pathway of citrinin in Monascus was reported by Blanc groups in 1995 [3]. Citrinin is a secondary metabolite produced by the late fermentation of Monascus which are usually produced at maturity or later stages of development, therefore, citrinin may be produced during Monascus production of Monascus Pigments. The biological metabolic pathway of citrinin produced by Monascus sp. is shown in Fig. 2 and Fig. 3 [4]

$\mathrm{Xu}$ Ganrong used TLC and HPLC to detect 40 strains of Monascus strains in yeast extract medium, and all the strains produced citrinin [5]. In addition, In addition, they also found with different concentrations of glutamic acid or aspartic acid as the sole carbon source, the concentration of glucose under the same conditions, with the increase of the concentration of nitrogen source, and the color value of citrinin content increased [6]. Lai Weihua research team pointed out that potato glucose medium is not conducive to citrinin production, however, yeast extract may promote the activity of citrinin synthase because of its rich nutrition and stimulate citrinin production by [7].

\subsection{Toxicity of Citrinin}

Early studies have shown that citrinin has antibacterial properties, inhibits bacterial reproduction, inhibits fungi, and inhibits protozoa [8]. Later, citrinin was found to have hepatotoxicity, reproductive toxicity, induction of cell aberration and apoptosis [9]. The target organ of citrinin is the kidney, and the toxicity is obvious. It can also cause teratogenicity, cause tumors and induce mutations. The median lethal dose of LD 50 was $110 \mathrm{mg} / \mathrm{kg}$ in mice [10], so Durackva et al think that the toxicity of citrinin is at the same level as aflatoxin B 1 [11]. Citrinin can make liver glycogen decreased, also can make the cholesterol and triglyceride synthesis blocked by inhibiting the synthesis of DNA, RNA, and proteins. In experiments with mice, citrinin can cause stable expression of genetically engineered cells to alter [12]. The Pfeiffer research team found that citrinin inhibits polymerase and leads to CREST- positive micronuclei in chromosomes of V 79 cells [13]. Cielger resarch group found that chicken embryos developing 4 days calls change after injection of citrinin [14]. Because of this toxic effect, citrinin has a certain antibacterial activity. It can inhibit Staphylococcus, Bacillus cereus, Bacillus cereus, Streptococcus aureus and some molds and yeasts. Wu Yu group found that citrinin toxin exposure inhibited mouse oocyte maturation and early embryo development [15]. Their study indicated that citrinin toxin exposure could reduce mouse oocyte maturation and early embryo development capability by affecting cytoskeletal dynamics, which may be due to the oxidative stress induced early apoptosis.

\subsection{Method for Detecting Citrinin}

The detection methods of citrinin are HPLC, TLC, enzyme linked immunosorbent assay, and bacteriostatic circle method, and so on. The lowest detection of citrinin limit $0.60 \mathrm{ng}$ was obtained by Chen Yun group using HPLC and fluorescence detection, and the linear relationship between the mass concentration of the standard citrinin solution and the peak area was good in the range of 0.1 to $10 \mathrm{mg} / \mathrm{L}$ mass concentration, $\mathrm{R}^{2}=0.998$ [16]. In addition, Chen Yun discussed the influence of experimental conditions on detection of citrinin and pointed out that when the retention time compared with acetonitrile as mobile phase citrinin peak tailing phenomenon serious, thereby affecting the accuracy of the qualitative and quantitative determination. Marley Elaine group established method performance characteristics using a citrinin immunoaffinity column (IAC) for sample cleanup before LC analysis with fluorescence detection (LC-fluorescence) to detect citrinin in various cereals and red yeast rice. The LOQ for citrinin in both wheat and red yeast rice was $10 \mu \mathrm{g} / \mathrm{kg}$, with an LOD of $3 \mu \mathrm{g} / \mathrm{kg}$ [17].

Thin layer chromatography (TLC) is commonly used for qualitative and semi quantitative determination of citrinin content in red yeast. It can be divided into unidirectional chromatography and bidirectional chromatography. In acidic aqueous solution as extraction agent, toluene: ethyl acetate: formic acid (6:3:1) as the agent, Hu Xiaoqing used TLC to isolate citrinin and obtained good separation effect using the difference between the water soluble pigment and fat soluble pigment dissolved in different solvents [18].

Enzyme linked immunosorbent assay (ELISA) is a new method based on the combination of antigen and antibody immune response and enzyme catalytic reaction. Direct competitive immunoassay could detect a buffer of $2 \sim 4 \mathrm{ng} / \mathrm{mL}$ citrinin, however, indirect competitive immunoassay could detect a buffer of 0.4 to $0.8 \mathrm{ng} / \mathrm{mL}$ citrinin. Abramson established an enzyme-linked immunosorbent assay for citrinin using conventional antiserum [19, 20]. But enzyme-linked immunosorbent assay is not suitable for widespread use because of the complexity of antibody preparation and high cost.

The method of bacteriostatic circle is relatively direct, 
which is qualitative and semi quantitative indirectly with the antimicrobial properties of citrinin. Citrinin has a strong inhibitory effect on Gram positive bacteria, which is most sensitive to Bacillus subtilis, and the size of its bacteriostatic circle correlates well with citrinin content. Monascus has many kinds of antibacterial substances, besides citrinin, it also has chitinase, orange pigment and Ankalactone and so on. Therefore, it is not accurate to detect citrinin in red yeast by bacteriostatic ring method.

In the above four kinds of citrinin detection methods, the method of bacteriostatic circle is simple to determine the amount of citrinin by the size of the bacteriostatic circle, but the results are not accurate enough. Thin layer chromatography is simple and easy, but there are many interference factors in the detection, and there are some problems in quantitative accuracy. Enzyme-linked immunosorbent assay (ELISA) is the most sensitive method at present, but the cost is too high. High performance liquid chromatography (HPLC) has high accuracy and reproducibility, and it is an accurate and reliable method.

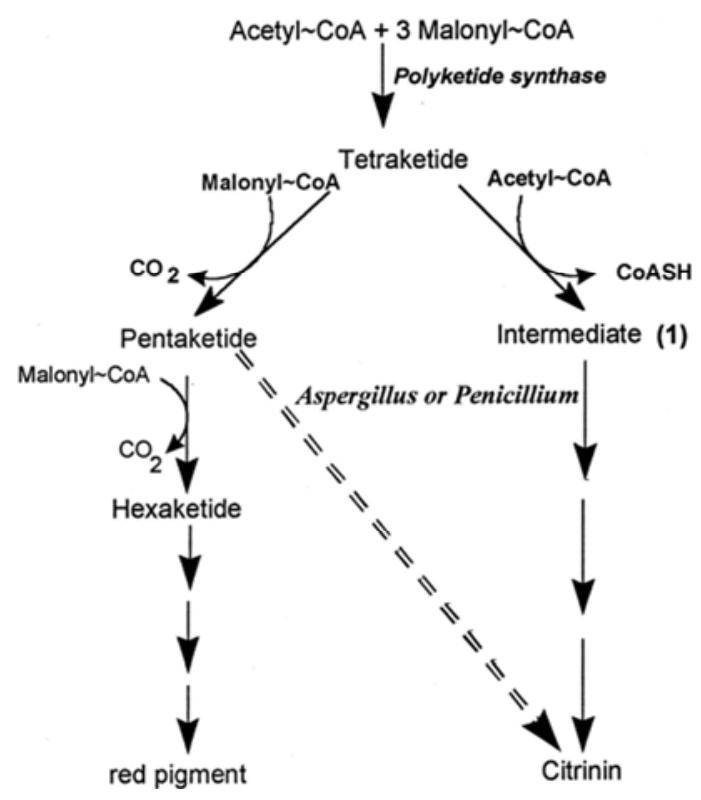

Figure 2. Biosynthesis of citrinin and red pigment in M. ruber.

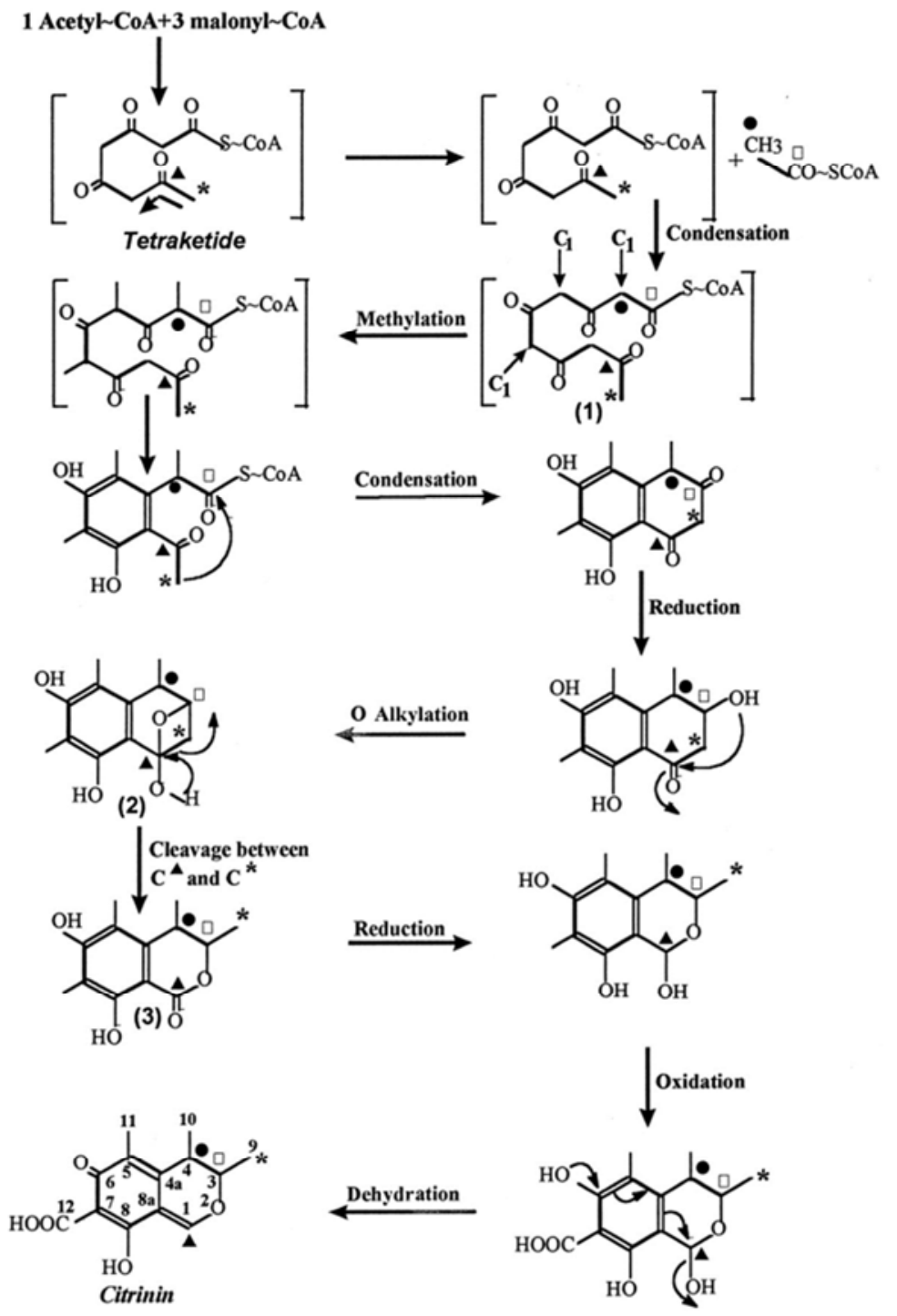

Figure 3. Scheme of the biosynthesis of citrinin by M. ruber. 


\subsection{Standard and Limits of Citrinin}

Citrinin has posed a severe challenge to the safety of Monascus products, which has attracted the attention of all countries. Japan formulated the limit of citrinin in Monascus pigment firstly. The limit index of citrinin in Monascus pigment was determined to be $0.2 \mathrm{mg} / \mathrm{kg}$ (color value standard was $500 \mathrm{U} / \mathrm{g}$ ), which was the lowest dose of citrinin that was detected at that time. The U.S. Food and drug administration has made it clear that Monascus products are recognized as food additives and must be evaluated for citrinin. In China's exports of Monascus products, Europe and the United States require the production of Monascus strains have been identified, and the citrinin content was limited strictly. Monascus imported from China must be certified for safe production, strains, and citrinin free in Germany.

In recent years, standards have been formulated for citrinin in Monascus products. "Determination of citrinin in Monascus products (GB/T 5009.222-2008)" was established in which the method for determination of citrinin in Monascus products was laied down, the limit of citrinin in liquid sample was 50 $\mathrm{g} / \mathrm{L}$ and the limit of citrinin in solid sample was $1 \mathrm{mg} / \mathrm{kg}$. In "National standard for food safety Standard for use of food additives GB 2760-2014", red yeast food additives include red kojic rice and Monascus red, and the CNS numbers are 8.119 and 8.120 respectively. They can be added as a colorant in 28 kinds of foods in which flavor fermented milk, cakes, baked foods, stuffing and surface pulp are specified for maximum use and other kinds of food need proper use according to production in addition. "National standard for food safety food additive-Monascus GB 1886.181-2016" was implemented in January 1, 2017 in which index requirements and inspection methods of citrinin in food additive Monascus red were specified. It's clear that the limit of citrinin (color unit price) is less than $0.04 \mathrm{mg} / \mathrm{kg}$ and the test method was determined according to GB5009.222.

\section{Detection and Analysis of Citrinin in Foods and Control Measures}

\subsection{Detection and Analysis of Citrinin in Foods}

HPLC method was used to determine citrinin content in several prepackaged foods purchased from supermarkets. The pretreatment of samples was carried out by ultrasonic assisted extraction centrifugal separation and nitrogen blowing concentration, and HPLC with fluorescence detection was used.

The chromatographic conditions are as follows (Table 1).

Table 1. Liquid chromatography condition.

\begin{tabular}{ll}
\hline Experimental Condition & Requirements \\
\hline chromatographic column & Agilent Eclipse Plus $\mathrm{C}_{18}$ \\
Column temperature & $28^{\circ} \mathrm{C}$ \\
Mobile phase & Acetonitrile: Water $=35: 65$ \\
Excitation wavelength & $\lambda \mathrm{ex}=331 \mathrm{~nm}$ \\
Emission wavelength & $\lambda \mathrm{em}=500 \mathrm{~nm}$ \\
Current Speed & $1 \mathrm{~mL} / \mathrm{min}$ \\
Sample size & $10 \mu \mathrm{L}$ \\
\hline
\end{tabular}

Commercially available Sufu (12 samples), cooked foods (15 samples), ham (23 samples) and snacks (7 samples) were studied on the content of citrinin. The results showed that citrinin was 23 positive and the positive rate was $40.4 \%$ (Below the detection limit is negative, opposite positive). The details are as follows (Table 2).

Table 2. The positive condition of citrinin in all kinds of foods.

\begin{tabular}{llllll}
\hline Samples & Sample number & Positive number & Positive rate (\%) & Citrinin Content range (mg/kg) & Average Content (mg/kg) \\
\hline Sufu & 12 & 11 & 91.7 & $0.096 \sim 0.24$ & 0.16 \\
cooked foods & 15 & 1 & 6.67 & 0.11 & 0.11 \\
ham & 23 & 8 & 34.8 & 0.123 & 0.19 \\
snacks & 7 & 3 & 42.8 & $0.096 \sim 0.24$ & 0.12 \\
total & 57 & 23 & 40.4 & 0.16 \\
\hline
\end{tabular}

Although the detection rate of citrinin in Sufu was the highest $(91.7 \%)$, the overall pollution level is not the highest. In $64 \%$ of the positive samples, citrinin levels were below 0.15 $\mathrm{mg} / \mathrm{kg}$.

\subsection{Measures for Controlling Citrinin in Monascus Products}

The importance of citrinin in Monascus production should be paid enough attention to red koji production industry. The fermentation technology of red kojic rice should be investigated systematically in order to produce Monascus products without citrinin or citrinin content.

In the process of production, strains with no citrinin or low content citrinin production should be screened, or the improvement of fermentation process to reduce citrinin production should be studied to formulate a reasonable fermentation process. Zhen-Qiang Ning group constructed a replacement-disrupted vector, and transformed the plasmid DNA into protoplasts of M. aurantiacus Li AS 3.4384, then obtained one ctnE disruptant to minimize citrinin production in Monascus fermentation by means of genetic engineering. This disruptant has greatly reduced citrinin production by $96 \%$ and increased pigment output by $40 \%$ compared to the wild-type [21]. In addition, physical and chemical degradation of citrinin could be used to solve citrinin in Monascus. Massimiliano Magro group proposed an efficient and reliable means for citrinin removal from Monascus treated foods using peculiar magnetic nanoparticles, synthesized in-house and called "Surface Active Maghemite Nanoparticles" [22].

These studies have given us a positive outlook for Monascus safety. With the development of new materials and 
technologies, and the application of gene technology and the improvement of production process, Monascus products without citrinin or citrinin minimum content (no harm to humans) will be producted.

\section{References}

[1] Srianta, I., Ristiarini, S., Nugerahani, I., Sen, S. K., Zhang, B. B., Xu, G. R., et al,. Recent research and development of Monascus fermentation products. Int. Food Res. J. 2014, 21 (1), $1-12$.

[2] Zhang XL, Wu TX, Li P. Dispute about the Safety of Citrinin of Monascus. Liquor-making Science \& Technology, 2007, (1), pp. $81-83$.

[3] P. J. Blanc, M. O. Loret, G. Goma. Production of citrinin by various species ofMonascus. Biotechnology Letters, March 1995, Volume 17, Issue 3, pp 291-294.

[4] Hajjaj, H; Klaebe, A; Loret, MO, et al. Biosynthetic Pathway of Citrinin in the Filamentous Fungus Monascus ruber as Revealed by 13 C Nuclear Magnetic Resonance. Applied and Environmental Microbiology, Jan. 1999, Volume 65, pp. 311-314.

[5] Xu GR, Chen Y., Gu YM, et al. Screening of Monascus Strains with Low Content Citrinin Production Ability. Journal of Wuxi University of Light Industry, 2000, 19 (1), pp. 58-61.

[6] Xu GR, Li FQ, Chen Y., et al. Monascus Citrinin Analysis Methods and a Study on Formation of Citrinin by Monascus. Microbiology, 2004, 31 (3), pp. 16-20.

[7] Lai WH, Xu Y., Li YP, et al. Study on Factors of Producing Citrinin by Monascus spp. China Brewing, 2004, (2), pp. 12-14.

[8] Biltrami K S, Sinha S P, Jeswal P. Proceeding of Indian National Science cademy, 1988, 54, pp. 35-37.

[9] Lu Y., Yuan H. the Review of Citrinin Cytotoxicity and its Detecton. China animal husbandry and veterinary medicine, 2012, 39 (1), pp. 60-63.

[10] Meng ZH, Zhang GZ, Song PJ. Mycotoxin Diagram [M]. Beijing: People's Medical Publishing House, 1979.

[11] VLADIMIR BETINA, CITRININ. Mycotoxin-Chemical [A]. Edited by Vladimir Betina. Biological and Environmental Aspects [C]. Elsevier: Amsterdam, 1989.
[12] Zhou Y., Hou HM. Research Advance of the antibiotie Substance from Monascus and Citinin [J]. Food Research and Development, 2005, 26 (1), pp. 20-22.

[13] Pfeiffer E, Gross K, Metzler M. Aneuploidogenic and clastogenic potential of the mycotoxins citrinin and patulin $[\mathrm{J}]$. Carcinogenic. 1998, 19, pp. 1313-1318.

[14] A Ciegler, R F Vesonder and L K Jackson. Produciton and biological activity of patulin and citrinin from Penicillium expansum [J]. Appl. and Environ. Microbiology, 1977, 33 (4), pp. 1004-1006.

[15] Yu Wu, Na Zhang, Ying-Hua Li, et al. Citrinin exposure affects oocyte maturation and embryo development by inducing oxidative stress-mediated apoptosis. Oncotarget, 2017, Vol. 8, (No. 21), pp: 34525-34533.

[16] Chen Y., Xu GR, Yu HL. Citrinin Analysis Using HPLC. Food and Fermentation Industries. 2004, 30 (1), pp. 118-123.

[17] Marley Elaine, Brown Phyllis, Leeman Dave, et al. Analysis of Citrinin in Cereals, Red Yeast Rice Dietary Supplement, and Animal Feed by Immunoaffinity Column Cleanup and LC with Fluorescence Detection. Journal of AOAC International, Volume 99, Number 4, 2016, pp. 1025-1031 (7).

[18] $\mathrm{Hu} X Q$, Chen FS, Xing SJ, et al. Thin layer chromatography analysis of citrinin in Monascus [J]. Food Science, 2003, 24 (5), pp. 126-129.

[19] Abramson D, Uslerber E, Artlbauer E M. An indirect enzyme immuncassay for the mycotoxin citrinin [J]. Applied and Environmental Microbiology. 1995, 61 (5), pp. 2007-2009.

[20] Abramson D. Determination of citrinin in barely by indirect and derect enzyme immuncassay [J]. J AOAC int. 1996, 79 (6), pp. 1325-1329.

[21] Zhen-Qiang Ning, Hua Cui, Yang $\mathrm{Xu}$, et al. Deleting the citrinin biosynthesis-related gene, ctnE, to greatly reduce citrinin production in Monascus aurantiacus Li AS 3.4384. International Journal of Food Microbiology, 2017, 241, pp. 325-330.

[22] Massimiliano Magro, Denise Esteves Moritz, Emanuela Bonaiuto, et al. Citrinin mycotoxin recognition and removal by naked magnetic Nanoparticles. Food Chemistry, 2016, 203, pp. $505-512$. 